\title{
EVALUACIÓN DE LA SITUACIÓN NUTRICIONAL Y ALIMENTARIA DE LOS NADADORES DE LA CATEGORÍA “SENIOR” DE LA SELECCIÓN NACIONAL DE COSTA RICA
}

\author{
${ }^{1}$ Jéssica Quesada González y ${ }^{2}$ María Mercedes Beltranena Falla de Enríquez \\ ${ }^{l}$ Escuela de Nutrición, Universidad de Costa Rica, San José, Costa Rica \\ ${ }^{2}$ Comité Olímpico Guatemalteco \\ E-mail: jequego@racsa.co.cr
}

\begin{abstract}
Resumen
Quesada González, J. y Beltranena Falla, M. M. (2002). Evaluación de la situación nutricional y alimentaria de los nadadores de la categoría "senior" de la Selección Nacional de Costa Rica. Revista de Ciencias del Ejercicio y la Salud, 2(2), 22-31. En años recientes se han llevado a cabo muchas investigaciones sobre el estado nutricional de diferentes grupos de población, sin embargo se desconocía la situación nutricional de los nadadores. El objetivo de este estudio fue evaluar la situación nutricional y alimentaria de los nadadores integrantes de la Selección Nacional en su categoría "senior", durante la etapa de entrenamiento, 1997. Fueron estudiados 16 deportistas en total, 9 hombres y 7 mujeres. Además de las mediciones antropométricas se valoraron sus indicadores bioquímicos sanguíneos, su alimentación fue evaluada mediante el método de registro diario de 3 días, su gasto energético se estimó a través del registro diario de actividades físicas Cuestionario de Bouchard. Los resultados mostraron porcentajes de grasa adecuados para ambos sexos, indicadores bioquímicos sanguíneos adecuados con excepción de las mujeres cuyos niveles de ferritina sérica se consideran bajos. Un $56 \%$ de los hombres consumen suplementos de vitaminas y minerales, la alimentación de todo el grupo es deficiente en fibra y alfatocoferol, ingieren megadosis de tiamina, riboflavina y piridoxina. Su balance energético es adecuado, no así la composición de la dieta ni la distribución energética en el día. Un $43 \%$ de las mujeres consumen suplementos de vitaminas y minerales, su alimentación no satisface las necesidades de fibra, calcio y niacina. Las mujeres que no consumen suplementos reportaron una dieta deficiente en fibra dietética, alfatocoferol, calcio, hierro y zinc. La totalidad de las mujeres presentan un balance de energía negativo, una composición de la dieta y una distribución energética diaria inadecuadas. Este estudio concluye que las mujeres no presentan un estado nutricional adecuado para una atleta y constituye una población a riesgo de sufrir la triada de problemas médicos que aquejan a la deportista. Los hombres, por el contrario, mantienen un estado nutricional adecuado lo cual favorece su rendimiento deportivo. PALABRAS CLAVES: Estado nutricional, natación, antropometría, ferritina sérica, dieta, indicadores bioquímicos.
\end{abstract}

\section{INTRODUCCION}

En el deporte de alto rendimiento la alimentación es, junto con las condiciones genéticas, el entrenamiento y la motivación, uno de los factores esenciales determinantes del rendimiento (Hamm, 1996). Sin embargo, el deterioro de esa capacidad de hacer ejercicio podría ser causado por carencias nutricionales que afectan el estado nutricional e inciden directamente sobre el desempeño deportivo del atleta (Beltranena, 1996).

La valoración del estado nutricional expresa el grado en el cual las necesidades fisiológicas de nutrientes que tiene el cuerpo humano, están siendo satisfechas. Sin embargo se debe considerar que el balance entre ingesta y requerimiento de nutrientes se ve influenciado por muchos factores tales como: hábitos alimentarios, enfermedad, crecimiento, infecciones, estrés fisiológico, 
socioeconómicos, entrenamiento fuerte $\mathrm{y}$ otros.

Según Plomden y Bernardot (1993), el entrenamiento pesado, como es el caso de aquel realizado por los nadadores, podría afectar negativamente la salud a través del aumento del riesgo de deshidratación y el incremento de las necesidades nutricionales que no están siendo cubiertas.

No todos los nadadores son conscientes de la importancia de mantener una alimentación acorde con sus necesidades, esta situación ha sido descrita por Berning (1992) en Estado Unidos, quien seleccionó como sujetos de investigación a 290 adolescentes, nadadores de elite, donde 152 y 138 de género masculino y femenino, respectivamente. En esta investigación se demostró, entre otras cosas, que solo un 67 $\%$ de las mujeres de la muestra y un $69 \%$ de los hombres, tenían conocimientos adecuados en nutrición.

Para analizar la dieta de un nadador es fundamental conocer no sólo la función de los nutrientes sino también los parámetros recomendados de estos nutrientes para una disciplina con las demandas fisiológicas y de nutrientes de esta disciplina deportiva.

Los carbohidratos ( $\mathrm{CHO})$ son una excelente fuente de energía, esencial para el ejercicio de moderada a alta intensidad como es el caso de la natación (Sierra, 1994) y son necesarios e importantes en las diferentes fases de la alimentación. Estos CHO se depositan o almacenan en nuestro cuerpo principalmente como glucógeno muscular y hepático, en cantidades limitadas, que en personas acondicionadas físicamente puede rondar entre 300 a 400 g (Sierra, 1994; Beltranena, 1996 y Creff y Berard, 1995). Además, Sherman, citado por Beltranena (1996), asegura que las reservas de glucógeno corporal dependen de la dieta, específicamente de la cantidad de $\mathrm{CHO}$ ingeridos y esto afectara el rendimiento deportivo los nadadores, pues la fatiga definida en términos estrictos, como la incapacidad de mantener la tasa de trabajo deseada (Wotton, 1990), se correlaciona con el agotamiento de las reservas de glucógeno y la consecuente falla para producir glucosa sanguínea para ser utilizada durante el ejercicio (Mahan y Escott-Stump, 1996).

Diferentes autores mencionan que con el fin de lograr la recuperación de las reservas de glucógeno es necesario que la dieta de los deportistas que se ejercitan intensamente, como es el caso de los nadadores, aporte entre un 60-70 \% del valor energético total (VET) como CHO (Plomden y Bernardot, 1993; Sierra, 1994; Beltranena, 1996; Grandjean, 1989).

En lo que grasas se refiere, como medida de prevención de enfermedad cardiovascular, varios autores coinciden en que la grasa total aportada por la dieta no debe pasar de un $30 \%$ del valor energético total y la ingesta de colesterol no debe ser mayor de $300 \mathrm{mg}$ al día (Beltranena, 1996; Murillo, 1996; Mahan y Escott-Stump, 1996; y Astrand, 1992). Por su parte, Konopka (1998) menciona que un nadador fondista y otro velocista deben consumir al menos de 25 y $30 \%$ del valor energético total, en forma de grasa, respectivamente.

Al hablar de proteínas, los requerimientos de este nutriente no pueden ser considerados aislados de la relación proteína - energía (Grandjean, 1989), pues la producción de energía a partir de proteína es la reacción que mayor cantidad de energía de oxigeno consume; además la importancia de este nutriente radica en su función de constructor muscular y no de combustible energético. Los entrenamientos de resistencia de alta intensidad conllevan siempre a un desgaste de las fibras musculares, así como a cambios estructurales en las membranas celulares y las mitocondrias, además de alteraciones en enzimas y hormonas; todo esto deriva una mayor ingesta de proteínas, que en la fase de regeneración tiene como resultado un incremento de la síntesis proteica y, por tanto, de la necesidad de ingerir proteína (Konopka, 1988).

Sin embargo, no sólo los macronutrientes deben ser valorados, pues 
diversas investigaciones en nutrición deportiva, han podido demostrar como puede afectarse la salud y el rendimiento deportivo de un atleta que sostenga por un periodo prolongado una dieta deficiente en vitaminas y minerales.

La composición corporal y los indicadores bioquímicas de un deportista son también parámetros que forman parte de la evaluación del esto nutricional de cualquier deportista y que deben ser, junto con la alimentación, mantenidos bajo estricta vigilancia para evitar que incidan de manera negativa sobre el rendimiento.

Soares, Ishii y Burini (1994), mencionan es su estudio con sujetos nadadores entre 15-26 años de edad, los hombres tenían una ingesta energética que oscilaba entre 4529 y $5493 \mathrm{kcal}$ con un aporte de CHO entre 543 y 674 g al día (VET aproximado de 48-49 \%). Mientras que las mujeres cuyas edades oscilaron entre 15 y 24 años mantuvieron ingestas energéticas entre 2669 y $3301 \mathrm{kcal}$, con un aporte de $\mathrm{CHO}$ de 298 a 374 g diarios correspondientes a un $45 \%$ del VET. En lo que a grasas se refiere, estos investigadores reportan ingestas en mujeres de 101-133 g. día $^{-1}$ (34-36 \% VET) y en hombres de 181$208 \mathrm{~g} \cdot \mathrm{dia}^{-1}(36-34 \%$ VET). Las proteínas fueron ingeridas en cantidades de $2.3-2.5 \mathrm{~g}$ $\cdot \mathrm{kg}^{-1} \cdot \mathrm{día}^{-1}$ en mujeres y de $2.6-3.3 \mathrm{~g} \cdot \mathrm{kg}^{-}$ ${ }^{1}$. día ${ }^{-1}$ en hombres.

Lukaski y cols. (1996), reportaron que en nadadores universitarios la ingesta promedio de $\mathrm{CHO}$ en mujeres y hombres fue de $260 \mathrm{~g}$ (50\% VET) y $440 \mathrm{~g}$ (45\% VET) diarios, respectivamente. Por otra parte la ingesta de grasa de los sujetos de este estudio fue de $142 \pm 9$ (32\% VET) para hombres y $87 \pm 3 \mathrm{~g} \cdot$ día para las mujeres (38 $\%$ VET). Además muestran una ingesta proteica de $1.2 \mathrm{~g} \cdot \mathrm{kg}^{-1}$. día ${ }^{-1}$ en mujeres y de $1.5 \mathrm{~g} \cdot \mathrm{kg}^{-1} \cdot \mathrm{día}^{-1}$ en hombres.

Por otra parte, Barr (1991), estudiando mujeres nadadoras universitarias encontró que ellas ingieren grasas en promedio $82 \pm 20 \mathrm{~g} \cdot$ día $^{-1}(32 \%$ VET), mientras que sus proteínas diarias corresponden a un promedio de $73 \pm 18 \mathrm{~g}$. día $^{-1}$ (32\% VET).

Dada la importancia de conocer el estado nutricional de los deportistas y considerando la escasa o inexistente información que se ha publicado a nivel nacional, el propósito de esta investigación fue evaluar la situación nutricional y alimentaria de los nadadores integrantes de la Selección Nacional en su categoría "senior", durante la etapa de entrenamiento, 1997.

\section{METODOLOGIA}

\section{Sujetos}

Nueve hombres con edades entre 15 y 23 años; siete mujeres entre 17-21 años; todos miembros de la Selección Nacional de Natación que participó en los Juegos Centroamericanos, en Honduras, 1997. Aunque 20 nadadores expresaron sus deseos de participar en el estudio, solo 16 cumplieron con todos los requisitos. Entrenan en promedio 22 horas a la semana, y el $94 \%$ de ellos realizan ejercicios de entrenamiento anaeróbico con pesas de 3 a 5 veces por semana durante 1 hora diaria. Esta investigación contó con la aprobación de la Federación Costarricense de Natación y Afines (FECONA) y la Escuela de Nutrición de la Universidad de Costa Rica. Además se obtuvo una autorización escrita de cada uno de los voluntarios antes de que participaran en la evaluación de su estado nutricional.

\section{Instrumentos de medición}

Composición corporal. Se determinó la talla, con una cinta métrica no extensible fijada en la pared y un cartabón. Para el peso corporal se utilizó una balanza calibrada (POMTREX, modelo 800-13159, capacidad máxima de $130 \mathrm{~kg}$ y sensibilidad 100 g). Con estas dos medidas de determinó el índice de masa corporal (IMC). 
Además, los pliegues cutáneos fueron determinados con un calibrador (HOLTAIN, sensibilidad de $0.2 \mathrm{~mm}$ ).

Indicadores

bioquímicos

sanguíneos. Las muestras de sangre fueron recolectadas siguiendo el protocolo estandarizado de una clínica privada en Costa Rica.

Calidad nutricional de la dieta. El consumo de alimentos se determinó mediante el uso de registro diario de consumo de 3 días (2 días entre semana y 1 día de fin de semana), para tal fin se utilizó el Diario de consumo (Chinnock, 1995). Para la determinación del valor nutricional de la dieta, la cantidad y el tipo de alimentos se codificó y procesó mediante un programa de cómputo denominado NUTRITIONIST $\mathrm{IV}^{\circledR}$. Las ingestas de suplementos dietéticos también fueron registradas.

Balance energético. Para poder estimar el gasto de energía, se utilizó un registro de actividad física llamado Cuestionario de Bouchard (Bouchard, 1983).

\section{Procedimientos}

Composición corporal. Se determinó la talla, sin zapatos ni medias, con una cinta métrica no extensible fijada en la pared y un cartabón. El peso corporal fue determinado antes del entrenamiento, los sujetos vestían únicamente sus trajes de baño.

Además se midieron 7 pliegues cutáneos (abdominal, biceps, triceps, muslo, subescapular, suprailíaco y pectoral, este último solamente en hombres. Todas estas mediciones se hicieron del lado derecho del cuerpo, por triplicado, registrándose el valor medio. El porcentaje de grasa corporal fue calculado a partir de la sumatoria de tres pliegues cutáneos según sexo y aplicando la ecuación de Jackson y Pollock en donde se obtuvo primero la densidad corporal y posteriormente se usó la ecuación de Siri (Aragón y Fernández, 1995).
Indicadores

bioquímicos

sanguíneos. Las muestras de sangre fueron recolectadas el mismo día que las medidas antropométricas, se determinó los valores de hemoglobina, hematocrito, CHCM, ferritina sérica, colesterol total, lipoproteínas de alta y baja densidad (HDL y LDL) y triglicéridos.

Calidad nutricional de la dieta. $\mathrm{El}$ consumo de alimentos se determinó una semana después de la recolección de datos antropométricos e indicadores bioquímicos sanguíneos, los nadadores recibieron la capacitación necesaria para llevar a cabo esta tarea. El mismo debía completarse de forma individual por cada sujeto.

Balance energético. Para poder estimar el gasto de energía, cada nadador completó durante los mismos 3 días del Diario de consumo, un registro de actividad física llamado Cuestionario de Bouchard (Bouchard, 1983), en donde anotó todas las actividades que realizó por períodos de 15 minutos.

\section{Análisis Estadístico}

Es un estudio descriptivo transversal. Se tomó a todos los nadadores del equipo y se recolectaron sus medidas antropométricas, indicadores bioquímicos sanguíneos y gasto energético, estos valores son presentados como promedios $(M)$ con sus desviaciones estándar $\left(\begin{array}{l} \pm \\ D S\end{array}\right)$, distribuidos según sexo. Datos de consumo de alimentos; se presentan como promedios y se aplicó también una prueba $t$-student, para comparar grupos. Estas variables fueron analizadas para determinar el estado nutricional de los sujetos.

\section{RESULTADOS}

Composición corporal. El cuadro 1 muestra los promedios respectivos a las mediciones antropométricas utilizadas, así como los indicadores que se derivan de estas. A partir de estos se observo que sujetos menores de 18 años tienen en promedio un IMC de 21.9 y 21.6 para los 
hombres y las mujeres respectivamente. Los nadadores mayores de 18 años tienen un IMC de 23 y $20.6 \mathrm{~kg} \cdot \mathrm{m}^{2}$ para hombres y mujeres respectivamente, lo cual es considerado normal (ACSM, 2000). El promedio de grasa corporal en nadadores menores de 18 años es de 5,6 \% y 17,8\% para hombres y mujeres respectivamente. Mientras que en los adultos se estimó en hombres $7.5 \%$ y en mujeres $16 \%$.

Cuadro 1. Características antropométricas $(M \pm D E)$, por sexo, durante la etapa de entrenamiento.

\begin{tabular}{lrr}
\hline \multicolumn{1}{c}{ Característica } & $\begin{array}{r}\text { Hombres } \\
(\mathbf{n = 9 )}\end{array}$ & $\begin{array}{r}\text { Mujeres } \\
(\mathbf{n = 7 )}\end{array}$ \\
\hline Peso $(\mathbf{k g})$ & $71.4 \pm 5.7$ & $59.7 \pm 3.1$ \\
Talla $(\mathbf{c m})$ & $178.5 \pm 5.2$ & $167.5 \pm 4.0$ \\
IMC $\left(\mathbf{k g} / \mathbf{m}^{2}\right)$ & $22.4 \pm 1.4$ & $21.3 \pm 1.2$ \\
Pliegues cutáneos & & \\
$\quad$ Bíceps $(\mathrm{mm})$ & $4.5 \pm 1.2$ & $6.3 \pm 2.1$ \\
$\quad$ Tríceps $(\mathrm{mm})$ & $6.3 \pm 1.7$ & - \\
Pectoral $(\mathrm{mm})$ & $6.1 \pm 1.7$ & $11.4 \pm 2.1$ \\
$\quad$ Subescapular $(\mathrm{mm})$ & $8.8 \pm 2.3$ & $10.9 \pm 3.3$ \\
Suprailíaco $(\mathrm{mm})$ & $6.6 \pm 1.2$ & $9.5 \pm 3.7$ \\
$\quad$ Abdominal $(\mathrm{mm})$ & $11.2 \pm 3.4$ & $14.1 \pm 4.1$ \\
$\quad$ Muslo $(\mathrm{mm})$ & $8.6 \pm 3.1$ & $20.6 \pm 5.8$ \\
\% de grasa corporal & $6.5 \pm 1.9$ & $17.0 \pm 3.3$ \\
\hline
\end{tabular}

Indicadores

bioquímicos

sanguíneos. En el cuadro 2 aparecen los promedios para los respectivos indicadores bioquímicos evaluados.

Calidad nutricional de la dieta.

Tanto en hombres como en mujeres se comprobó la utilización de suplementos de vitaminas y minerales tales como: Centrum, Supradyn, Vitamina C, Megamen y Ultramega, dado el aporte nutricional de estos productos fue necesaria la división de estos grupos. Para ambos sexos, el grupo 1 incluye a los sujetos que no consumían suplementos, mientras que el grupo 2, agrupa aquellos que sí los consumen. De acuerdo con los datos, 5 de los 9 hombres $(56 \%)$ toman suplementos; y 3 de las 7 mujeres $(43 \%)$ también realizan esta práctica.
El cuadro 3 muestra que hubo varias diferencias significativas entre ambos grupos de mujeres, tal es el caso de las proteínas, vitamina A, alfa tocoferol, vitamina $\mathrm{C}$, tiamina, rivoflabina, piridoxina, cobre, hierro y zinc. El caso de los hombres fue distinto, pues solo demostraron diferencias significativas para la viatmina A y la vitamina $\mathrm{C}$.

Cuadro 2. Estadística descriptiva $(M \pm D E)$ de los valores bioquímicos recolectados durante la etapa de entrenamiento.

\begin{tabular}{lcc}
\hline Parámetro bioquímico & $\begin{array}{r}\text { Hombres } \\
(\mathbf{n = 9 )}\end{array}$ & $\begin{array}{r}\text { Mujeres } \\
(\mathbf{n = 7})\end{array}$ \\
\hline Perfil lipídico & & \\
$\quad$ Colesterol total (mg/dl) & $149.8 \pm 22.1$ & $169.7 \pm 23.7$ \\
HDL (mg/dl) & $42.6 \pm 8.9$ & $54.0 \pm 4.5$ \\
LDL (mg/dl) & $93.3 \pm 17.8$ & $105.1 \pm 22.5$ \\
Triglicéridos (mg/dl) & $72.1 \pm 37.7$ & $52.6 \pm 39.3$ \\
Cuadro hematologico & & \\
$\quad$ Hemoglobina (g/dl) & $16.0 \pm 0.7$ & $14.5 \pm 0.8$ \\
Hematocrito (\%) & $48 \pm 3$ & $43.7 \pm 2.2$ \\
CHCM (\%) & $33.4 \pm 0.6$ & $33.1 \pm 0.8$ \\
Ferritina sérica (ng/ml) & $178.0 \pm 25.2$ & $45.0 \pm 12.8$ \\
\hline
\end{tabular}

La distribución porcentual de macronutrientes al valor energético total es presentada en el Cuadro 5, observándose una distribución de $\mathrm{CHO}$, proteínas y grasas de: 54.7; 15.9; $29.4 \%$ (hombres); 59.3; 13.0; $27.5 \%$ (grupo 1 mujeres); 62.3; 10.3 y 27.3 $\%$ (grupo 2 mujeres).

El gráfico 1 muestra que el patrón alimentario presenta una distribución de 5 a 7 tiempos de comida para ambos sexos, de manera que el desayuno, la merienda de la mañana, el almuerzo, la merienda de la tarde y la cena fueron tiempos de comida realizados por la totalidad de la muestra. $\mathrm{La}$ diferencia se presenta en las meriendas antes del desayuno y la nocturna, en donde no todos los sujetos consumen algún alimento a estas horas.

Balance energético. Según el cuestionario de Bouchard los hombres tienen un gasto energético promedio de 3599 
kilocalorías \pm 531 , y las mujeres 2861 kilocalorías \pm 287 . Estos datos demuestran que durante los días del estudio, la adecuación del balance de energía en hombres fue de $92 \%$, mientras que en mujeres fue de $77 \%$.

Cuadro 3. Valor Nutritivo $(M \pm D E)$ de la dieta de las mujeres nadadoras de la Selección Nacional de Costa Rica $(n=7)$. Valores presentados según consumo de suplementos.

\begin{tabular}{lrrr}
\hline \multicolumn{1}{c}{ Nutriente } & Ingesta total & Grupo 1 $(\boldsymbol{n}=\mathbf{4})$ & Grupo 2 $(\boldsymbol{n}=\mathbf{3})$ \\
\hline Energía (kcal) & $2205 \pm 286$ & $2246 \pm 104$ & $2150 \pm 384$ \\
Carbohidratos totales(g) & $346.0 \pm 83.9$ & $342.7 \pm 40.6$ & $350.5 \pm 11.4$ \\
Azúcar (g) & $137.0 \pm 80.5$ & $115.4 \pm 13.1$ & $165.7 \pm 130.5$ \\
$\quad$ Fibra dietética (g) & $13 \pm 3$ & $12 \pm 3$ & $13 \pm 14$ \\
Proteínas (g) & $66.0 \pm 12.5$ & $74.7 \pm 8.2^{*}$ & $54.6 \pm 3.7^{*}$ \\
Grasa (g) & $67.8 \pm 10.0$ & $70.5 \pm 10.8$ & $64.1 \pm 7.7$ \\
Colesterol (mg) & $244 \pm 108$ & $244 \pm 109$ & $245 \pm 106$ \\
Vitamina A (mcg RE) & $1883 \pm 1292$ & $900 \pm 274^{*}$ & $3193 \pm 509^{*}$ \\
Alfa-tocoferol (mg ET) & $14.5 \pm 11.2$ & $5.6 \pm 0.6^{*}$ & $26.4 \pm 1.5^{*}$ \\
Vitamina C (mg) & $507 \pm 495$ & $202 \pm 122^{*}$ & $915 \pm 430^{*}$ \\
Tiamina (mg) & $2.34 \pm 0.89$ & $1.69 \pm 0.27^{*}$ & $3.22 \pm 0.39^{*}$ \\
Rivoflabina (mg) & $2.40 \pm 0.80$ & $1.78 \pm 0.23^{*}$ & $3.24 \pm 0.13^{*}$ \\
Niacina (mg) & $17.65 \pm 4.36$ & $19.09 \pm 5.08$ & $15.73 \pm 2.39$ \\
Piridoxina (mg) & $2.47 \pm 1.15$ & $1.68 \pm 0.12^{*}$ & $3.53 \pm 0.80^{*}$ \\
Calcio (mg) & $751 \pm 222$ & $796 \pm 302$ & $692 \pm 34$ \\
Fósforo (mg) & $1078 \pm 199$ & $1142 \pm 254$ & $993 \pm 48$ \\
Potasio (mg) & $2767 \pm 978$ & $2609 \pm 410$ & $2977 \pm 1292$ \\
Magnesio (mg) & $270 \pm 82$ & $279 \pm 89$ & $257 \pm 72$ \\
Hierro (mg) & $21.60 \pm 9.78$ & $14.16 \pm 3.20^{*}$ & $31.53 \pm 3.56^{*}$ \\
Cobre (mg) & $2.16 \pm 1.08$ & $1.33 \pm 0.26^{*}$ & $3.27 \pm 0.33^{*}$ \\
Zinc (mg) & $12.83 \pm 7.45$ & $6.91 \pm 1.17^{*}$ & $20.72 \pm 0.90^{*}$ \\
\hline Na & \pm & &
\end{tabular}

Nota: Grupo 1: Dieta sin suplementos; Grupo 2: Dieta con suplementos.

$* p<0.05$ entre grupos

\section{DISCUSIÓN}

El IMC encontrado en los sujetos menores de 18 años (21.9 y 21.6 para los hombres y las mujeres respectivamente), los ubica entre los percentiles 25 y 75 según los datos suministrados por CDC (2000), los clasifica como adolescentes con un peso normal. Los nadadores mayores de 18 años (IMC de 23 y $20.6 \mathrm{~g} / \mathrm{m}^{2}$ para hombres y mujeres, respectivamente), tenían un peso considerado normal para su talla (ACSM, 2000). El promedio de grasa corporal en nadadores menores de 18 años es de $5.6 \%$ y $17.8 \%$ para hombres y mujeres respectivamente. Mientras que en los adultos se estimó en hombres $7.5 \%$ y en mujeres $16 \%$. Todos los valores obtenidos se consideran normales para esta disciplina deportiva.

Sin embargo, los resultados antropométricos observados difieren de aquellos encontrados en otras investigaciones. Así, por ejemplo, en hombres el porcentaje de grasa es menor que el encontrado en otros estudios: $10.8-10.9$ $\%$ (Lukaski y cols., 1996); y $10.9 \%$ (Soares, Ishii, y Burini, 1994.)

Por el contrario, las nadadoras presentan porcentajes de grasa mayores que los encontrados por estos investigadores: $12.6-12.7 \%$ (Lukaski y cols., 1996); y 15 
\% (Soares, Ishii, y Burini, 1994), pero menores que los reportados por Barr (1991):
$21.9 \%$. Para todos los grupos los resultados son adecuados (Houtkooper y Going, 1994).

Cuadro 4. Valor nutritivo $(M \pm D E)$ de la dieta de los hombres nadadores de la Selección Nacional de Costa Rica $(n=9)$. Valores presentados según consumo de suplementos.

\begin{tabular}{|c|c|c|c|}
\hline Nutriente & Ingesta total & Grupo $1(n=4)$ & Grupo $2(n=5)$ \\
\hline Energía (kcal) & $3309 \pm 1065$ & $3127 \pm 1175$ & $3454 \pm 1085$ \\
\hline Carbohidratos totales(g) & $447.8 \pm 119.4$ & $436.5 \pm 113.8$ & $456.7 \pm 136.3$ \\
\hline Azúcar (g) & $163.5 \pm 55.4$ & $131.1 \pm 43.3$ & $189.5 \pm 53.4$ \\
\hline Fibra dietética (g) & $13 \pm 6$ & $14 \pm 5$ & $13 \pm 8$ \\
\hline Proteínas (g) & $132.2 \pm 49.7$ & $126.2 \pm 62.8$ & $137.0 \pm 43.7$ \\
\hline Grasa (g) & $113.7 \pm 54.8$ & $100.1 \pm 58.0$ & $124.7 \pm 54.9$ \\
\hline Colesterol (mg) & $554 \pm 248$ & $424 \pm 283$ & $657 \pm 162$ \\
\hline Vitamina A (mcg RE) & $2339 \pm 1447$ & $1184 \pm 736^{*}$ & $3264 \pm 1145^{*}$ \\
\hline Alfa-tocoferol (mg ET) & $31.2 \pm 47.3$ & $5.2 \pm 2.2$ & $52.0 \pm 57.1$ \\
\hline Vitamina C (mg) & $363 \pm 209$ & $177 \pm 93^{*}$ & $513 \pm 127^{*}$ \\
\hline Tiamina (mg) & $29.06 \pm 66.39$ & $2.16 \pm 0.85$ & $50.58 \pm 86.68$ \\
\hline Rivoflabina (mg) & $18.56 \pm 33.53$ & $2.44 \pm 0.91$ & $31.46 \pm 42.18$ \\
\hline Niacina (mg) & $43.67 \pm 43.57$ & $25.02 \pm 11.68$ & $58.60 \pm 55.16$ \\
\hline Piridoxina (mg) & $40.73 \pm 99.46$ & $2.18 \pm 0.90$ & $71.57 \pm 130.80$ \\
\hline Calcio (mg) & $1256 \pm 686$ & $995 \pm 363$ & $1464 \pm 843$ \\
\hline Fósforo (mg) & $2049 \pm 728$ & $1840 \pm 620$ & $2217 \pm 826$ \\
\hline Potasio (mg) & $3948 \pm 1649$ & $3340 \pm 764$ & $4435 \pm 2087$ \\
\hline Magnesio (mg) & $457 \pm 153$ & $412 \pm 92$ & $493 \pm 187$ \\
\hline Hierro (mg) & $21.07 \pm 9.90$ & $17.18 \pm 8.50$ & $19.47 \pm 2.89$ \\
\hline Cobre (mg) & $2.08 \pm 1.01$ & $1.75 \pm 0.62$ & $2.34 \pm 1.21$ \\
\hline Zinc (mg) & $22.94 \pm 19.19$ & $14.85 \pm 8.79$ & $29.41 \pm 23.40$ \\
\hline
\end{tabular}

Nota: Grupo 1: Dieta sin suplementos; Grupo 2: Dieta con suplementos.

$* p<0.05$ entre grupos

En el Cuadro No. 2 se observa que todos los sujetos mantienen los indicadores dentro de los rangos considerados normales para la población en general (Clinica Krebs, noviembre 1997). La hemoglobina de las mujeres se mantiene en el rango superior de la normalidad, sin embargo, los niveles de ferritina sérica, aunque son adecuados, se ubican dentro de los valores mínimos del rango $(11-307 \mathrm{ng} / \mathrm{ml})$. En los indicadores bioquímicos sanguíneos encontrados las mujeres presenten una ferritina sérica baja, por tanto ellas podrían ubicarse en el primer estadío de anemia ferropriva.

Al hablar de calidad de la dieta, según las ingestas de nutrientes reportadas, se determinó que en hombres, con excepción de la fibra (43\%), todos los porcentajes exceden el $100 \%$ de la recomendación de nutrientes dada por INCAP y OPS (1994). La ingesta de tiamina excede la recomendación 16 veces $(1614 \%)$, la riboflavina 11 veces $(1031 \%)$ y la piridoxina 28 veces $(2798 \%)$.

Las mujeres del grupo 1 alcanzan porcentajes de adecuación menores al $100 \%$ en fibra dietética (40\%), alfa-tocoferol (70 $\%)$, calcio $(80 \%)$, hierro (87\%) y zinc (77 $\%)$; en el grupo 2 en estas mismas circunstancias se tiene la fibra dietética (43 $\%$ ), calcio (69\%) y magnesio (96\%), por el contrario, la Vitamina $\mathrm{C}$ tiene un porcentaje de adecuación de $1525 \%$. 
Por tanto, después de este análisis es importante destacar que los sujetos utilizan suplementos de vitaminas y minerales como complemento de la dieta, por ejemplo aquellos que se adquieren en farmacia y que brinda dosis de vitaminas y minerales más proporcionadas (Centrum, Vitamina C, Supradin y Vitaminas del complejo B) o aquellos suplementos que se dicen naturales o artificiales y que son promocionados por comercio y gimnasios (Megamen y Ultramega). Estos últimos cubren de 2 a 20 veces las recomendaciones dietéticas, dependiendo del nutriente.

Cuadro 5. Contribución porcentual de macronutrientes al valor energético total de la dieta según sexo. Etapa de entrenamiento.

\begin{tabular}{lccc}
\hline \multirow{2}{*}{ Macronutrientes } & \multirow{2}{*}{ Hombres (\% VET) } & \multicolumn{2}{c}{ Mujeres (\% VET) } \\
\cline { 3 - 4 } & & Grupo 1 & Grupo 2 \\
\hline Carbohidratos totales & $54.7 \pm 6.6$ & $59.3 \pm 5.0$ & $62.3 \pm 10.7$ \\
$\quad$ Azúcar* & $20.3 \pm 5.1$ & $20.5 \pm 1.7$ & $28.3 \pm 17.9$ \\
Proteínas & $15.9 \pm 49.7$ & $13.0 \pm 1.6$ & $10.3 \pm 4.5^{* *}$ \\
Grasas & $29.4 \pm 6.3$ & $27.5 \pm 4.4$ & $27.3 \pm 7.4$ \\
\hline
\end{tabular}

Nota: * Los porcentajes de este carbohidrato simple forman parte del que se da para los carbohidratos totales.

$* * p<0.05$ entre grupo 1 (dieta sin suplementos) y grupo 2 (dieta con suplementos)

La adecuación de la dieta entre los grupos de mujeres muestra que el grupo 1 mantiene una dieta deficiente en fibra, alfatocoferol, hierro, calcio y zinc, mientras que el grupo 2 reporta una alimentación deficiente en fibra y calcio. El grupo de hombres, satisface en promedio todas sus necesidades de nutrientes, con excepción de la fibra dietética, lo cual es el reflejo de un inadecuado consumo de frutas y vegetales, característico de la población costarricense. Los hombres exceden la recomendación de colesterol, lo cual podría representar un riesgo futuro de enfermedad cardiovascular. Para la tiamina y la riboflavina la ingesta promedio del grupo de hombres es respectivamente de 16 y 10 veces más de la recomendada, su requerimiento es fácil de llenar mediante una dieta en la que los alimentos sean densos en nutrientes y que no proporcionen únicamente calorías vacías, por esta razón no es necesario suplementarlas. La ingesta de piridoxina es 28 veces mayor a la recomendación, la literatura reporta que altas dosis de esta vitamina, tomada durante meses o años, podría causar daño nervioso irreversible (Whitney, Cataldo, y Rolfes, 1994).
Las mujeres del grupo 2 muestran porcentajes de adecuación para la Vitamina C, 15 veces mayores a la recomendación, y la literatura reporta que en altas dosis (10 veces la recomendación dietética) tiene efectos tóxicos como nauseas, espasmos abdominales y diarrea (Whitney, Cataldo, y Rolfes, 1994). Ninguno de los dos grupos de mujeres satisface la recomendación de calcio, comprometiendo la salud ósea, esto es parte de la triada de problemas médicos que aquejan a muchas deportistas. El mineral hierro, no es ingerido en cantidades adecuada por el grupo 1, y aunque el grupo 2 sobrepasa la recomendación, la biodisponibilidad de esta ingesta de hierro podría no ser la mejor. Eso se refleja en los bajos niveles de ferritina sérica que manejan las mujeres. El zinc es el otro mineral reportado como deficiente en la dieta del grupo 1 de mujeres.

Ni los hombres ni los dos grupos de mujeres tienen una distribución adecuada de macronutrientes, se destaca el alto porcentaje de energía que es aportado por $\mathrm{CHO}$ simples (azúcar), donde el máximo recomendado es de $10 \%$ del valor energético total. 
La dieta de los hombres es rica en grasa y proteínas, baja en $\mathrm{CHO}$, lo cual según hace que no se tenga el combustible adecuado ni en calidad ni en cantidad para que un deportista lleve a cabo una ejecución adecuada. Al mismo tiempo, una dieta rica en grasa requiere la conversión de aminoácidos a glucosa.

Los hombres del estudio derivan proporcionalmente mayor cantidad de energía de los $\mathrm{CHO}(54.7 \%)$ menor cantidad de las grasas $(29.4 \%)$ en comparación con los nadadores otros estudios (Lukaski, y cols., 1996; Soares, Ishii, y Burini, 1994).

En lo que a proteínas se refiere, los hombres consumen $1.9 \mathrm{~g} / \mathrm{kg}$ de peso/día, valor que sobrepasa lo recomendado para la etapa de entrenamiento $(1.5 \mathrm{~g} / \mathrm{kg} / \mathrm{día})$ (Williams, 1995), superando el $15 \%$ del VET para estos deportistas.

En las mujeres se encontró que el grupo 2 de mujeres ingiere significativamente menos proteína que el grupo 1 debido a que una vez analizadas sus dietas de manera cualitativa se pudo observar que consumen menores cantidades de carne, productos lácteos y huevos. Por su lado, el grupo 1 tampoco presenta valores adecuados en la distribución de proteínas.

$\mathrm{El}$ alto porcentaje obtenido de $\mathrm{CHO}$ simples sugiere que una suficiente cantidad de alimentos consumidos por los nadadores les aportan únicamente calorías vacías y no son alimentos de alto valor nutritivo.

En los hombres se encontró de acuerdo con el porcentaje de adecuación entre ingesta y gasto que los nadadores están consumiendo la energía suficiente para cubrir el gasto energético, mientras que las mujeres tienen un porcentaje de adecuación del $77 \%$, el cual se encuentra muy por debajo del rango de normalidad (90-110\%). Este es uno de limitantes del desarrollo de masa muscular, pues dada la baja ingesta de nutrientes, hasta las proteínas deben ser utilizadas en mayor grado para la obtención de energía.

Gráfico 1. Distribución porcentual de la energía diaria consumida por tiempo de comida.

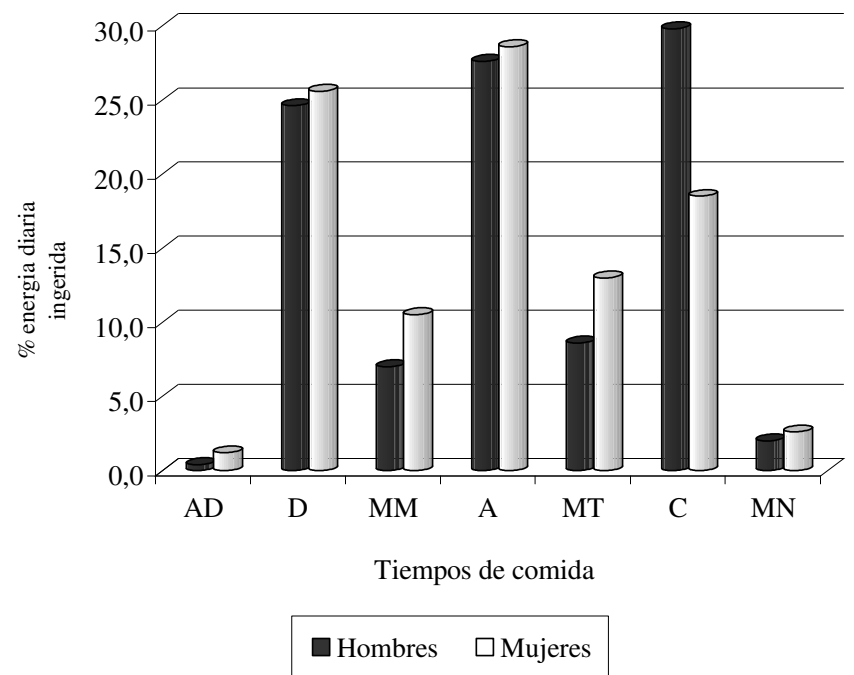

Leyenda: $\mathrm{AD}=$ merienda antes del desayuno $; \mathrm{D}=$ desayuno; $\mathrm{MM}=$ merienda de la mañana $; \mathrm{A}=$ almuerzo $; \mathrm{MT}=$ merienda de la tarde; $\mathrm{C}=$ cena; $\mathrm{MN}=$ merienda nocturna. El desayuno aporta una menor
cantidad de energía que aquella
recomendada (20-40\% VET) (Beltranena,
1996; Sierra, 1994), esto podría ser causado por su horario de estudio. El almuerzo mantiene los lineamientos sugeridos (27\%), 
siendo ésta la comida más importante para el grupo de mujeres. La cena tiene una distribución diferente, pues las mujeres consumen una cantidad de energía que tiende a ser menor que en el caso de los hombres, para ellos ésta se convierte en la máxima ingesta energética durante el día. De hecho, algunas mujeres tratan de hacer de la cena una merienda, que solamente les quite un poco la sensación de hambre. En las meriendas las mujeres tienden a consumir mayor energía que los hombres, lo cual es beneficioso si se recuerda que tanto la merienda antes del desayuno como la de la tarde se realizan antes del entrenamiento y por consiguiente, contribuye con el mantenimiento del suministro constante de glucosa a las células musculares, necesario para el desarrollo de los diferentes ejercicios. Normalmente, los sujetos consumen alimentos dentro de las 2 horas posteriores al ejercicio, lo cual contribuye con la reposición del glucógeno muscular. Sin embargo, las mujeres no consumen suficiente cantidad de alimento en la cena que les permita reponer satisfactoriamente ese glucógeno.

Las mujeres en su mayoría están arriesgando un poco por conseguir el alto rendimiento, pues podrían estar experimentando una triada de problemas médicos. Dos de los problemas de esta triada son claramente identificados en las nadadoras de este estudio, pues se relacionan con la salud del hueso y desórdenes alimentarios. El estado nutricional de las mujeres nadadoras de la categoría "senior" no es el óptimo para una deportista, pues aunque sus indicadores antropométricos se clasifican como adecuados, sus indicadores bioquímicos evidencian deficiencia de hierro y la calidad nutricional de la dieta muestra deficiencias importantes.

El estado nutricional de los hombres nadadores es adecuado para un deportista, tal $\mathrm{y}$ como lo evidencian sus indicadores antropométricos, bioquímicos y dietéticos. No obstante, se deben controlar los excesos en la ingesta de algunos nutrientes debido al uso indiscriminado de suplementos, que lejos de contribuir con su rendimiento deportivo, podría perjudicar la salud del nadador.

\section{REFERENCIAS}

Aragón, LF y Fernández A. (1995). Fisiología del Ejercicio: respuestas entrenamiento $y$ medición. San José, Costa Rica. Editorial de la Universidad de Costa Rica. pp. 266 - 281.

Barr, S. I. (1991). Relationship of eating attitudes to anthropometric variables and dietary intakes of females colllegiate swimmers. Journal of the American Dietetic Association, 91(8), 976-977.

Beltranena, M. M. (1996). Alimentación del deportista. Capacitación para entrenadores de alto rendimiento, Diciembre 1996. Guatemala. sp.

Bouchard, C. (1983). A method to assess energy expenditure in children and adults. American Journal of Clinical Nutrition, 37(3), 461-467.

Chinnock, A. (1995). Diario de consumo de Alimentos. Escuela de Nutrición e Instituto de Investigaciones en Salud. Universidad de Costa Rica. San José, Costa Rica.

Houtkooper, L. B y Going, S. B. (1994). Body composition: how should it be measured? Does it affect sport performance?. Sports Science Exchange, 7(5), sp.

Konopka, P. (1988). La alimentación del deportista. México, D.F.: Ediciones Martínez Roca.

Lukaski, H. C. et al. (1996). Iron, copper, magnesium and zinc status as predictors of swimming performance. International Journal of Sports Medicine, 17(Sn), 535-540.

First Databank Division. (1995). Nutritionist IV: Food Product and Nutrition Labeling. Versión 4.0.

Plomden, M. y Bernardot, D. (1993). Position of the American Dietetic Association and the Canadian Dietetic Association: Nutrition for physical fitness and athletic performance for adults. Journal of the American Dietetic Association, 93(6), 691-696.

Soares, E.A., Ishii, M. y Burini, R. (1994). Estudo antropométrico e dietético de nadadores competitivos de áreas metropolitanas da região sudeste do Brasil. Rev. Saúde Pública, 28(1), 919.

Sierra, E. (1994). Manual de Nutrición Deportiva. Bogotá, Colombia: Universidad Nacional de Colombia.

Whitney, E., Cataldo, C. y Rolfes, S. (1994). Understanding Normal and Clinical Nutrition ( $4^{\text {th }}$ Ed.). Minneapolis, USA: West Publishing Company

Williams, M. H. (1995). Nutrition for fitness and sport. $\left(4^{\text {th }}\right.$ Ed.). New York, NY: Brown and Benchmark Publishers. Pp. 7-17, 46-48. 